\title{
Tumor-infiltrating lymphocyte subsets and tertiary lymphoid structures in pulmonary metastases from colorectal cancer
}

\author{
Thomas Schweiger ${ }^{1,2}$ Anna Sophie Berghoff ${ }^{3,4}$ Christoph Glogner ${ }^{1,2}$. \\ Olaf Glueck $^{1,2}$ - Orsolya Rajky ${ }^{3,4}$ - Denise Traxler ${ }^{1,2} \cdot$ Peter Birner $^{5}$. \\ Matthias Preusser $^{3,4} \cdot$ Walter Klepetko ${ }^{1} \cdot$ Konrad Hoetzenecker $^{1}$
}

Received: 21 February 2016/ Accepted: 18 July 2016/Published online: 23 July 2016

(C) The Author(s) 2016. This article is published with open access at Springerlink.com

\begin{abstract}
The presence of tumor-infiltrating lymphocytes (TILs) and tertiary lymphoid structures (TLSs) reflects an active inflammatory tumor microenvironment. High density of TILs as well as presence of TLS is associated with improved survival in various solid cancer types. We aimed to describe the density and distribution of TILs and TLS in pulmonary metastases (PMs) from primary colorectal cancer (CRC) and its correlation with clinicopathological variables. Fifty-seven CRC pulmonary metastasectomy specimen (PM) and 31 matched primary CRC specimen were included. Cluster of differentiation (CD) $3+, \mathrm{CD} 8+$, $\mathrm{CD} 45 \mathrm{RO}+$ and FoxP3+ TILs were evaluated by immunohistochemistry and density was scored semiquantitatively. TLS were evaluated based on morphological criteria. Survival time was defined from pulmonary metastasectomy to death or last follow up. A marked
\end{abstract}

Electronic supplementary material The online version of this article (doi:10.1007/s10585-016-9813-y) contains supplementary material, which is available to authorized users.

Konrad Hoetzenecker

konrad.hoetzenecker@meduniwien.ac.at

1 Division of Thoracic Surgery, Department of Thoracic Surgery, Medical University of Vienna, Waehringer Guertel 18-20, 1090 Vienna, Austria

2 Christian Doppler Laboratory for Cardiac and Thoracic Diagnosis and Regeneration, Medical University of Vienna, Vienna, Austria

3 Department of Medicine I, Medical University of Vienna, Vienna, Austria

4 Comprehensive Cancer Center, Medical University of Vienna, Vienna, Austria

5 Department of Pathology, Medical University of Vienna, Vienna, Austria infiltration with $\mathrm{CD} 3+, \mathrm{CD} 8+, \mathrm{CD} 45 \mathrm{RO}+$ and FoxP3+ TILs was evident in CRC PM and matched primary CRC. Further assessment of the immune infiltrate in PM showed that a high density of FOXP3 + TILs at the invasive margin [HR $2.40(1.11-6.96) ; P=0.031]$ and low density of CD8+ cells in TLS [HR $0.30(0.14-0.79) ; P=0.016$ ] were associated with a worse prognosis in univariate analysis. Moreover, a low CD8/FoxP3-ratio of TILs at the invasive margin $(P=0.042)$ and in TLS $(P=0.027)$ conferred an impaired prognosis after pulmonary metastasectomy. Our findings suggest that CRC PM harbor an immune active microenvironment. The balance of CD8+ and FoxP3 + T-cells at the tumor border and in TLS provides prognostic information in patients with CRC PM.

Keywords Pulmonary metastasis - Colorectal cancer . Tumor-infiltrating lymphocytes $\cdot$ TILs $\cdot$ Tertiary lymphoid structures

\section{Background}

Despite advances in the early detection and treatment of colorectal cancer (CRC), the prognosis of patients is impaired as soon as distant metastases occur. Synchronous pulmonary spreading is evident in about one out of ten patients with newly diagnosed CRC. Subsequently, an average 5-year cumulative risk of $5.8 \%$ for the development of metachronous pulmonary metastases (PMs) is additionally contributing to the disease burden of patients with CRC [1]. However, within the group of patients with CRC lung metastases long-term survival can be achieved by (repeated) pulmonary metastasectomy complemented by chemotherapeutic regimens. In contrast, some patients will present with diffuse recurrence of disease within months after pulmonary 
metastasectomy. The underlying tumor biology is considered to be the main cause for heterogeneity in the outcome of patients with CRC lung metastases. Several prognostic biomarkers have been proposed to define aggressive tumors associated with fatal outcome [2-4]. During the last years, the tumor microenvironment gained increasing attention in the scientific community, especially in groups focusing on metastatic CRC [5-7].

Immune escape is considered an emerging hallmark of cancer [8]. Various subsets of lymphocytes can be found in the tumor microenvironment, so called tumor-infiltrating lymphocytes (TILs). They can launch pro-inflammatory anti-tumor responses or mediate local immunosuppression. The amount of TILs has a prognostic value in various primary solid cancer types, including lung, renal, breast and CRC [9-14]. Commonly detected lymphocyte subsets with favorable prognostic impact are mature T-cells [cluster of differentiation (CD)3+] and cytotoxic T-cells $(\mathrm{CD} 8+)$, memory-T-cells (CD45RO+), while immune suppressive regulatory $\mathrm{T}$-cells (FoxP3+) are associated with impaired prognosis. Moreover, tertiary lymphoid structures (TLSs), which are ectopic lymphoid aggregates present in chronically inflamed tissue, can be found in the tumor stroma. TLS are believed to promote and maintain inflammation and anti-tumor response similar to secondary lymphoid organs. The presence of TLS in the tumor microenvironment is associated with favorable prognosis especially in CRC $[15,16]$.

So far little is known about the local immune response in CRC metastases, as previous studies focused on primary tumor specimen. Metastasis initiating cells have already successfully conquered immune escape during intravasation, survival in the blood stream and extravasation indicating that the composition of the immune microenvironment might differ from the primary tumor $[17,18]$. Therefore, we aimed to investigate the local inflammatory microenvironment in CRC PM specimen and matched primary CRC specimen.

\section{Materials and methods}

\section{Study population}

From April 2009 to June 201457 patients with primary CRC receiving complete/curative pulmonary metastasectomy at the Medical University of Vienna were retrospectively recruited from a prospective institutional database. The study was approved by the Institutional Ethics Committee (\# 1035/2014) and conducted according the Declaration of Helsinki. Resected pulmonary nodules were verified as metastases from primary CRC by a board certified pathologist. Additionally, samples of the corresponding primary tumor were available in $31 / 57$ $(54.4 \%)$ patients. The patients underwent post-surgical tumor surveillance after pulmonary metastasectomy including periodical computed tomography (CT) scans.

\section{Immunohistochemistry}

Immunohistochemical (IHC) staining was performed on $4 \mu \mathrm{m}$ thick sections of formalin-fixed, paraffin-embedded tissue samples using an automated staining platform (Ventana Benchmark Ultra immunostainer, Ventana Medical Systems, Inc., Tucson, USA).

Immunostaining was performed with anti-CD3 (clone SP7, \#RM9107-S1, Thermo Fisher Scientific, Cheshire, UK), anti-CD8 (clone C8/144B, \#M7103, Dako, Glostrup, Denmark), anti-CD45RO (clone UCHL1, \#M074201, Dako, Glostrup, Denmark) and anti-FOXP3 (clone 206D, \#320116, BioLegend, San Diego, CA, USA) antibodies using an autostainer (Benchmark Ultra, Ventana Medical Systems, Tucson, USA) according to the manufacturer's instructions. In negative controls the primary antibody was omitted. A mediastinal lymph node served as positive control. The presence of lymphatic vessel invasion in PM was known from a previous study [19].

\section{Quantification and scoring of TILs and TLS}

The density of CD3+, CD8+, CD45RO+ and FoxP3+ TILs was evaluated in a semiquantitative manner on full size sections as described previously [20, 21]. In summary, scores reaching from 0 (absent infiltrate) to $4+$ (very dense infiltrate) were assigned to the tissue samples. First, an overall impression was rated at low magnification $(\times 100)$. Additionally, the spatial distribution of the immune infiltrate was assessed in the tumor center and at the invasive margin separately at higher magnification $(\times 200-400)$. For further analyses, the TILs densities were dichotomized. The used cut-offs are provided in Supplementary Table 1. Moreover, the sections were screened for the presence of TLSs based on morphologic features (TLS; present vs. not present). Follicular aggregates of lymphatic cells were defined as TLS, whereas perivascular lymphatic aggregates were excluded. The presence of $\mathrm{CD} 3+, \mathrm{CD} 8+, \mathrm{CD} 45 \mathrm{RO}+$ and FoxP3+ cells in the TLS was assessed and, according to TILs quantification, scores from 0 to 4 were assigned to the samples. In general, negative and sparse infiltration $(0$ and $1+)$ were grouped in contrast to moderate, dense and very dense infiltration $(2+, 3+$ and $4+)$, which led to two group sizes as equal as possible. As CD3 were omnipresent, a higher cut-off had to be applied. Also for CD8 TILs, a higher cut off was chosen as described previously by others $[22,23]$. The dichotomized variables were used for further calculations. CD8/FoxP3 ratios were calculated 
from dichotomized values. Samples with high CD8/low FoxP3 were described as CD8/FoxP3-ratio high, samples with high $\mathrm{CD} 8 /$ high FoxP3 or low CD8/low FoxP3 as equal and low CD8/high FoxP3 were described as low. Two observers (TS, ASB) blinded to the clinical data rated each section independently using a multi-head microscope. If the rating differed, the slide was re-discussed and a consensus was found.

\section{Statistical analysis}

The disease-free interval (DFI) was defined as the time between surgery for the primary tumor to pulmonary metastasectomy in months. Time to recurrence represented the time between pulmonary metastasectomy and evidence of recurrence at any organ site. Overall survival (OS) was defined as the period of time between pulmonary metastasectomy and death of any cause. If patients had a history of pulmonary metastasectomy before the inclusion period, the previously resected metastases were assessed and the outcome was calculated from the first pulmonary metastasectomy. Nominal variables were compared using $\chi^{2}$ test or Fisher's exact test (if expected frequency $<5$ ). Survival curves were estimated using Kaplan-Meier plots and the difference of the groups were compared using the log-rank test. Statistically significant variables $(P$-values $\leq 0.05)$ in the univariate analysis were added to a multivariate Cox regression model. Statistics were performed using SPSS 23 (SPSS, Inc., Chicago, USA) and GraphPad Prism 6 (GraphPad Software, Inc., California, USA) software. All performed tests were two-sided. $P$-values $\leq 0.05$ were considered statistically significant. Due to the hypothesis generating approach of the study no correction for multiple testing was used [24].

\section{Results}

Fifty-seven patients with histologically verified PMs from primary CRC were included in this study. Macroscopically and microscopically complete resection was achieved in all patients. $33(57.9 \%)$ patients were male and $24(42.1 \%)$ female. Median age at the time of pulmonary metastasectomy was 64 years (range 33-83). The primary tumor site was colon in $32(56 \%)$ patients and rectum in $25(44 \%)$ patients. A detailed description of the patients' characteristics is provided in Table 1.

\section{Density and distribution of TILs and TLS in pulmonary metastases}

Sufficient IHC quality was achieved in 55/57 (96.5\%), $57 / 57$ (100\%), 54/57 (94.7\%) and 53/57 (93.0\%) of PM specimen for $\mathrm{CD} 3+, \mathrm{CD} 8+, \mathrm{CD} 45 \mathrm{RO}+$ and FoxP3+ TIL evaluation, respectively. $\mathrm{CD} 3+, \mathrm{CD} 8+, \mathrm{CD} 45 \mathrm{RO}+$ and FoxP3 + TILs at variable density were evident in 55/55 $(100 \%), 55 / 57$ (96.5\%), 50/54 (92.3\%) and 45/53 $(84.9 \%)$ of PM. A detailed description of the density and spatial distribution of TILs is shown in Table 2. Representative images of TILs are provided in Fig. 1. TLS were present in 45/57 (78.9\%) PM specimen. If TLS could be found, the density of $\mathrm{CD} 3+, \mathrm{CD} 8+, \mathrm{CD} 45 \mathrm{RO}+$ and FoxP3 + T-cells in TLS was assessed (Supplementary Table 2) and correlated with clinicopathological characteristics (Supplementary Table 3).

The density of CD3+, CD8+, CD45RO+ and FoxP3+ TILs was correlated with clinicopathological characteristics of our patients. Intratumoral FoxP3 + TILs were more often present in patients with single $\mathrm{PM}\left(\chi^{2} P=0.011\right)$. All (10/10 (100\%) patients with a DFI of more than 60 months had high levels of CD45RO+ TILs at the invasive margin, compared to $24 / 36(66.6 \%)$ patients in the group DFI $<36$ months (DFI $>60$ vs. $<36$ months; Fisher's exact test $P=0.044)$. FoxP3 + TILs at the invasive margin were associated with evidence for lymphatic vessel invasion $\left(\chi^{2}\right.$ test $P=0.050)$. High CD3 + infiltration in the tumor center was more often found in PM from colon cancer compared to rectal cancer $\left(\chi^{2} P=0.040\right)$. Otherwise, no association of TILs density with age, sex, tumor localization, UICC stage of the primary tumor, previous liver metastasis or chemotherapy before metastasectomy was evident (Table 3).

\section{Correlation of TILs and TLS in pulmonary metastases and corresponding primary CRC}

A detailed description of $\mathrm{CD} 3+, \mathrm{CD} 8+, \mathrm{CD} 45 \mathrm{RO}+$ and FoxP3+ TILs density in the tumor center and at the invasive margin of the corresponding primary tumors is provided in Supplementary Table 4. We found no significant correlation between the TIL density in the primary tumor and corresponding lung metastases (Supplementary Table 5). In general, PM had higher densities of $\mathrm{CD} 3+, \mathrm{CD} 8+$, CD45RO+ TILs, whereas the FoxP3 TIL were comparable (Supplementary Fig. 1). Significantly less primary CRC were rated as TLS positive compared to the paired PM samples [2/28 (7.1\%) vs. 22/28 (78.6\%); McNemar test $P<0.001]$.

\section{Impact of TILs and TLS in PM on outcome parameters}

The presence of FoxP3 + TILs at the invasive margin was significantly associated with a decreased OS (35 vs. 65 months; HR 2.40; $95 \%$ CI 1.11-6.96; log-rank test $P=0.031$ ) (Fig. 2). Moreover, dense CD8+ infiltrates at the invasive margin were associated with improved OS 
Table 1 Demographic details of the study cohort $(n=57)$

\begin{tabular}{|c|c|c|}
\hline \multirow[t]{2}{*}{ Characteristics } & \multicolumn{2}{|c|}{$\begin{array}{l}\text { Total study cohort } \\
(\mathrm{n}=57)\end{array}$} \\
\hline & $\mathrm{n}$ & $\%$ \\
\hline Median age at surgery (range) & $64(33-83)$ & \\
\hline Median follow-up after metastasectomy in months (range) & $30(4-137)$ & \\
\hline \multicolumn{3}{|l|}{ Sex } \\
\hline Male & 33 & 57.9 \\
\hline Female & 24 & 42.1 \\
\hline \multicolumn{3}{|l|}{ Localization of primary tumor } \\
\hline Colon & 32 & 56.1 \\
\hline Rectum & 25 & 43.9 \\
\hline \multicolumn{3}{|l|}{ UICC stage of primary tumor } \\
\hline I & 4 & 7.4 \\
\hline II & 14 & 25.9 \\
\hline III & 27 & 50.0 \\
\hline IV & 9 & 16.7 \\
\hline Unknown & 3 & - \\
\hline \multicolumn{3}{|l|}{ Previous liver metastasis } \\
\hline Yes & 17 & 29.8 \\
\hline No & 40 & 70.2 \\
\hline \multicolumn{3}{|l|}{ DFI } \\
\hline$<36$ months & 38 & 66.7 \\
\hline $36-60$ months & 9 & 15.8 \\
\hline 60 months & 10 & 17.5 \\
\hline \multicolumn{3}{|l|}{ No. of pulmonary metastases } \\
\hline Singular & 37 & 64.9 \\
\hline Multiple & 20 & 35.1 \\
\hline \multicolumn{3}{|l|}{ Chemotherapy before metastasectomy } \\
\hline Yes & 45 & 78.9 \\
\hline No & 12 & 21.1 \\
\hline \multicolumn{3}{|l|}{ Chemotherapy after metastasectomy } \\
\hline Yes & 43 & 75.4 \\
\hline No & 14 & 24.6 \\
\hline
\end{tabular}

DFI disease-free survival to first pulmonary metastasis (median survival not reached vs. 39 months; HR 0.00; $95 \%$ CI 0.09-1.04; log-rank test $P=0.064$ ).

6/29 (20.7\%) patients had a high CD8/FoxP3-ratio, CD8/FoxP3 was equal in 10/29 (34.5\%) patients and a low ratio was found in $13 / 29$ (44.8\%) patients. The CD8/ FoxP3-ratio had significant impact on OS prognosis after pulmonary metastasectomy (log-rank test $P=0.021$ and 0.042 for low vs. equal and low vs. high, respectively). Furthermore, patients with a high CD8/FoxP3-ratio at the invasive margin had a prolonged recurrence-free survival after pulmonary metastasectomy compared to patients with low CD8/FOXP3 ratio (26 vs. 15 months; HR 0.32; $95 \%$ CI 0.12-1.03; log-rank test $P=0.080$ ).

The presence of TLSs in PM alone was neither associated with recurrence-free survival (log-rank test
$P=0.141)$ nor with OS $(P=0.813)$. However, when assessing the T cell subsets of TLS, we found a significant association of high levels of CD8+ cells in TLS and improved OS (median survival not reached vs. 35 months; HR 0.30; $95 \%$ CI 0.14-0.79; log-rank test $P=0.016$ ). Similarly to TILs, a strong prognostic effect was also found for the CD8/FOXP3-ratio in TLS (high vs. low HR 6.99; $95 \%$ CI 1.28-20.48; log-rank test $P=0.027)$. Outcome analyses of $\mathrm{CD} 3+, \mathrm{CD} 8+, \mathrm{CD} 45 \mathrm{RO}+$ and FoxP3+ cells in TLS are summarized in Table 4 and Fig. 3. Adding $\mathrm{CD} 8+$ in TLS and FoxP3 + at the invasive margin into a multivariate Cox regression model, only CD8+ cells in TLS remained a significant prognosticator for OS after pulmonary metastasectomy $(P=0.020$; HR $0.29 ; 95 \% \mathrm{CI}$ $0.07-0.79)$. 
Table 2 Semi-quantitative description of tumor-infiltrating lymphocytes in CRC pulmonary metastases

\begin{tabular}{|c|c|c|c|c|}
\hline \multirow[t]{2}{*}{ TILs density } & CD3+ TILs & CD8+ TILs & CD45RO+ TILs & FoxP3+ TILs \\
\hline & $\%$ & $\%$ & $\%$ & $\%$ \\
\hline
\end{tabular}

Tumor center

$\begin{array}{lrrrrrrrr}0 & 0 & 0.0 & 2 & 3.5 & 4 & 7.0 & 8 & 14.0 \\ 1+ & 7 & 12.3 & 28 & 49.1 & 18 & 31.6 & 19 & 33.3 \\ 2+ & 21 & 36.8 & 15 & 26.3 & 28 & 49.1 & 24 & 42.1 \\ 3+ & 20 & 35.1 & 12 & 21.1 & 4 & 7.0 & 2 & 3.5 \\ 4+ & 7 & 12.3 & 0 & 0.0 & 0 & 0.0 & 0 & 0.0 \\ \text { Total } & 55 & 96.5 & 57 & 100.0 & 57 & 100.0 & 57 & 100.0\end{array}$

Invasive margin

\begin{tabular}{lrrrrrrrr}
0 & 0 & 0.0 & 13 & 22.8 & 2 & 3.5 & 10 & 17.5 \\
$1+$ & 3 & 5.3 & 21 & 36.8 & 13 & 22.8 & 26 & 45.6 \\
$2+$ & 20 & 35.1 & 17 & 29.8 & 32 & 56.1 & 17 & 29.8 \\
$3+$ & 22 & 38.6 & 6 & 10.5 & 7 & 12.3 & 0 & 0.0 \\
$4+$ & 10 & 17.5 & 0 & 0.0 & 0 & 0.0 & 0 & 0.0 \\
Total & 55 & 96.5 & 57 & 100.0 & 54 & 94.7 & 53 & 93.0 \\
\hline
\end{tabular}
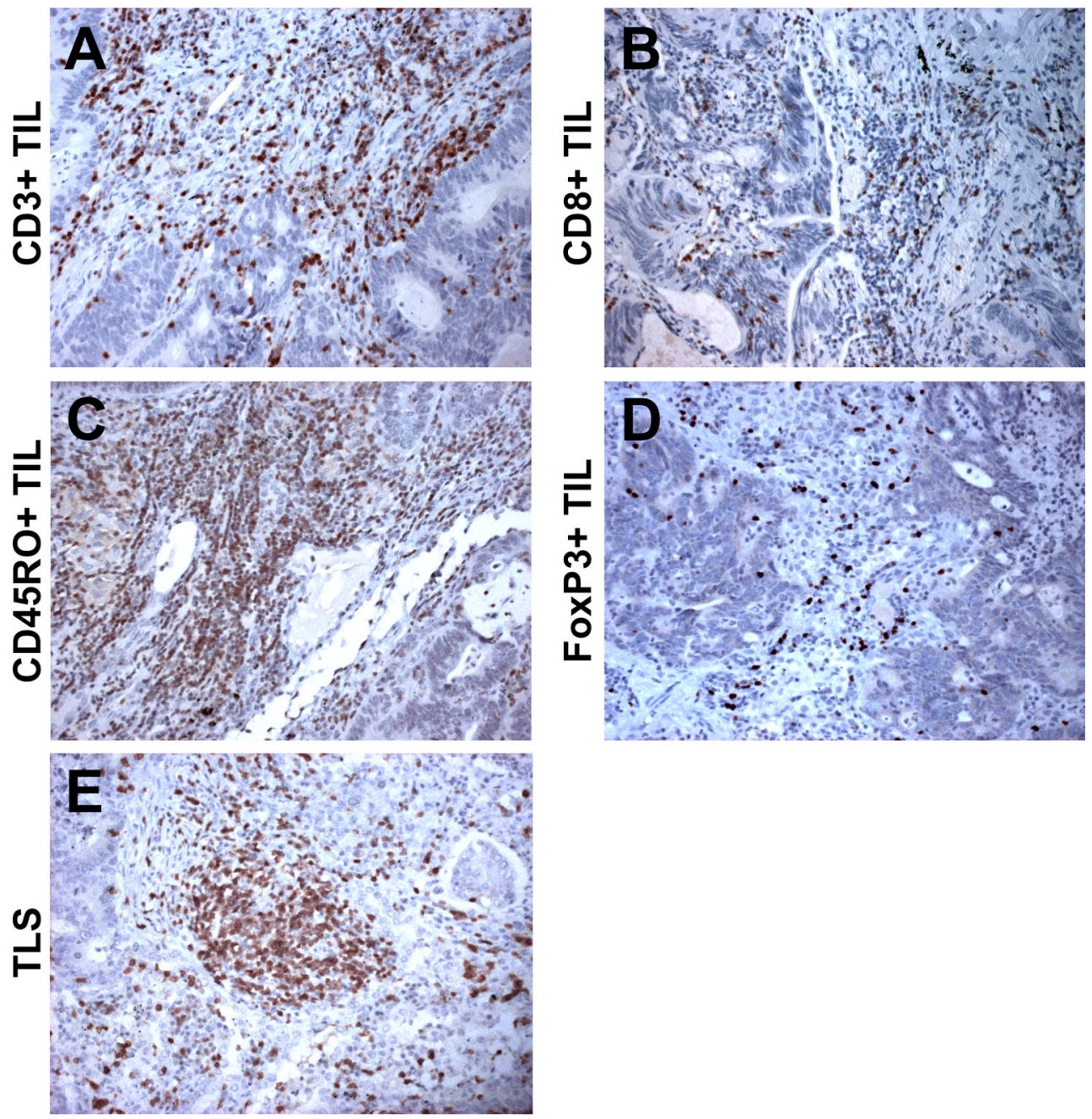

Fig. 1 Representative images of high densities of a CD3+, b CD8+, c CD45RO+ and $\mathbf{d}$ FoxP3+ TILs (DAB; brown) in pulmonary metastases. e CD3+ tertiary lymphoid structure at the invasive margin between tumor cells and lung parenchyma (magnification $\times 200 / 400)$. $($ Color figure online) 
Table 3 Association of CD3+, CD8+, CD45RO+ and FoxP3+ TILs in the tumor center and at the invasive margin with clinicopathological characteristics (significant values $(P<0.05)$ in bold)

\begin{tabular}{|c|c|c|c|c|c|c|c|c|c|c|c|c|}
\hline & \multicolumn{6}{|c|}{ CD3+ TILs } & \multicolumn{6}{|c|}{ CD8+ TILs } \\
\hline & \multicolumn{3}{|c|}{ Tumor center } & \multicolumn{3}{|c|}{ Invasive margin } & \multicolumn{3}{|c|}{ Tumor center } & \multicolumn{3}{|c|}{ Invasive margin } \\
\hline & Low & High & $P$ & Low & High & $P$ & Low & High & $P$ & Low & High & $P$ \\
\hline \multicolumn{13}{|l|}{ Age at surgery } \\
\hline$<64$ & 12 & 15 & 0.346 & 9 & 18 & 0.210 & 21 & 7 & 0.473 & 24 & 4 & 0.423 \\
\hline$\geq 64$ & 16 & 12 & & 14 & 14 & & 24 & 5 & & 27 & 2 & \\
\hline \multicolumn{13}{|l|}{ Sex } \\
\hline Male & 15 & 17 & 0.480 & 12 & 20 & 0.444 & 24 & 9 & 0.177 & 29 & 4 & 1.000 \\
\hline Female & 13 & 10 & & 11 & 12 & & 21 & 3 & & 22 & 2 & \\
\hline \multicolumn{13}{|c|}{ Localization of primary tumor } \\
\hline Colon & 12 & 19 & 0.040 & 13 & 18 & 0.984 & 23 & 9 & 0.138 & 28 & 4 & 0.686 \\
\hline Rectum & 16 & 8 & & 10 & 14 & & 22 & 3 & & 23 & 2 & \\
\hline \multicolumn{13}{|c|}{ UICC stage of primary tumor } \\
\hline $\mathrm{I}$ & 1 & 3 & 0.815 & 0 & 4 & 0.165 & 3 & 1 & 0.511 & 3 & 1 & 0.292 \\
\hline II & 6 & 6 & & 4 & 8 & & 13 & 1 & & 14 & 0 & \\
\hline III & 15 & 12 & & 15 & 12 & & 20 & 7 & & 23 & 4 & \\
\hline IV & 4 & 5 & & 3 & 6 & & 7 & 2 & & 8 & 1 & \\
\hline \multicolumn{13}{|c|}{ Unknown $(\mathrm{n}=3)$} \\
\hline \multicolumn{13}{|c|}{ Previous liver metastasis } \\
\hline No & 17 & 22 & 0.090 & 18 & 21 & 0.309 & 30 & 10 & 0.315 & 35 & 5 & 0.657 \\
\hline Yes & 11 & 5 & & 5 & 11 & & 15 & 2 & & 16 & 1 & \\
\hline \multicolumn{13}{|l|}{ DFI } \\
\hline$<36$ months & 21 & 17 & 0.547 & 18 & 20 & 0.343 & 32 & 6 & 0.271 & 35 & 3 & 0.554 \\
\hline $36-60$ months & 4 & 4 & & 3 & 5 & & 7 & 2 & & 8 & 1 & \\
\hline 60 months & 3 & 6 & & 2 & 7 & & 6 & 4 & & 8 & 2 & \\
\hline \multicolumn{13}{|c|}{ No. of pulmonary metastases } \\
\hline Singular & 17 & 18 & 0.646 & 13 & 22 & 0.352 & 29 & 8 & 1.000 & 32 & 5 & 0.410 \\
\hline Multiple & 11 & 9 & & 10 & 10 & & 16 & 4 & & 19 & 1 & \\
\hline \multicolumn{13}{|c|}{ Lymphatic vessel invasion } \\
\hline No & 20 & 14 & 0.135 & 15 & 19 & 0.660 & 29 & 6 & 0.506 & 31 & 4 & 1.000 \\
\hline Yes & 8 & 13 & & 8 & 13 & & 16 & 6 & & 20 & 2 & \\
\hline \multicolumn{13}{|c|}{ Chemotherapy before metastasectomy } \\
\hline No & 4 & 7 & 0.281 & 3 & 8 & 0.326 & 10 & 2 & 1.000 & 11 & 1 & 1.000 \\
\hline \multirow[t]{4}{*}{ Yes } & 24 & 20 & & 20 & 24 & & 35 & 10 & & 40 & 5 & \\
\hline & \multicolumn{6}{|c|}{ CD45RO+ TILs } & \multicolumn{6}{|c|}{ FoxP3+ TILs } \\
\hline & \multicolumn{3}{|c|}{ Tumor center } & Invas & margin & & Tumo & enter & & Invasi & margin & \\
\hline & Low & High & $P$ & Low & High & $P$ & Low & High & $P$ & Low & High & $P$ \\
\hline Age at surgery & & & & & & & & & & & & \\
\hline$<64$ & 10 & 17 & 0.580 & 6 & 21 & 0.362 & 15 & 12 & 0.494 & 19 & 8 & 0.697 \\
\hline$\geq 64$ & 12 & 15 & & 9 & 18 & & 12 & 14 & & 17 & 9 & \\
\hline Sex & & & & & & & & & & & & \\
\hline Male & 11 & 20 & 0.361 & 10 & 21 & 0.393 & 16 & 15 & 0.908 & 22 & 9 & 0.573 \\
\hline Female & 11 & 12 & & 5 & 18 & & 11 & 11 & & 14 & 8 & \\
\hline Localization of $\mathrm{p}$ & tumor & & & & & & & & & & & \\
\hline Colon & 13 & 8 & 0.836 & 11 & 20 & 0.142 & 16 & 15 & 0.908 & 22 & 9 & 0.573 \\
\hline Rectum & 9 & 14 & & 4 & 19 & & 11 & 11 & & 14 & 8 & \\
\hline
\end{tabular}


Table 3 continued

\begin{tabular}{|c|c|c|c|c|c|c|c|c|c|c|c|c|}
\hline & \multicolumn{6}{|c|}{ CD45RO+ TILs } & \multicolumn{6}{|c|}{ FoxP3+ TILs } \\
\hline & \multicolumn{3}{|c|}{ Tumor center } & \multicolumn{3}{|c|}{ Invasive margin } & \multicolumn{3}{|c|}{ Tumor center } & \multicolumn{3}{|c|}{ Invasive margin } \\
\hline & Low & High & $P$ & Low & High & $P$ & Low & High & $P$ & Low & High & $P$ \\
\hline \multicolumn{13}{|c|}{ UICC stage of primary tumor } \\
\hline I & 2 & 2 & 0.891 & 0 & 4 & 0.453 & 1 & 3 & 0.228 & 3 & 1 & 0.393 \\
\hline II & 4 & 9 & & 2 & 11 & & 4 & 8 & & 6 & 6 & \\
\hline III & 11 & 15 & & 8 & 18 & & 17 & 9 & & 20 & 6 & \\
\hline IV & 3 & 5 & & 3 & 5 & & 4 & 4 & & 6 & 2 & \\
\hline \multicolumn{13}{|c|}{ Unknown $(\mathrm{n}=3)$} \\
\hline \multicolumn{13}{|c|}{ Previous liver metastasis } \\
\hline No & 14 & 24 & 0.369 & 9 & 29 & 0.333 & 16 & 21 & 0.088 & 23 & 14 & 0.172 \\
\hline Yes & 8 & 8 & & 6 & 10 & & 11 & 5 & & 13 & 3 & \\
\hline \multicolumn{13}{|l|}{ DFI } \\
\hline$<36$ months & 17 & 19 & 0.298 & 12 & 24 & 0.082 & 19 & 18 & 0.641 & 24 & 13 & 0.547 \\
\hline $36-60$ months & 3 & 5 & & 3 & 5 & & 5 & 3 & & 7 & 1 & \\
\hline 60 months & 2 & 8 & & 0 & 10 & & 3 & 5 & & 5 & 3 & \\
\hline \multicolumn{13}{|c|}{ No. of pulmonary metastases } \\
\hline Singular & 15 & 21 & 0.845 & 10 & 26 & 1.000 & 14 & 22 & 0.011 & 22 & 14 & 0.122 \\
\hline Multiple & 7 & 11 & & 5 & 13 & & 13 & 4 & & 14 & 3 & \\
\hline \multicolumn{13}{|c|}{ Lymphatic vessel invasion } \\
\hline No & 14 & 19 & 0.752 & 11 & 22 & 0.253 & 17 & 15 & 0.695 & 25 & 7 & $\mathbf{0 . 0 5 0}$ \\
\hline Yes & 8 & 13 & & 4 & 17 & & 10 & 11 & & 11 & 10 & \\
\hline \multicolumn{13}{|c|}{ Chemotherapy before metastasectomy } \\
\hline No & 6 & 6 & 0.517 & 3 & 9 & 1.000 & 4 & 7 & 0.277 & 7 & 4 & 0.730 \\
\hline Yes & 16 & 26 & & 12 & 30 & & 23 & 19 & & 29 & 13 & \\
\hline
\end{tabular}

DFI disease-free survival to first pulmonary metastasis

\section{Discussion}

The aim of this study was to evaluate the role of TILs and TLSs in PM assessing a cohort of patients with CRC lung metastases. CD3+ TILs were found in every resected pulmonary metastatic specimen, highlighting the pivotal role of the adaptive immune system in local tumor microenvironment. We could show that tumor infiltrating $\mathrm{CD} 8+$ and FoxP3 positive cells were associated with disease free survival after pulmonary metastasectomy and OS.

$\mathrm{CD} 8+\mathrm{T}$ cells represent a subpopulation of $\mathrm{T}$ cells, also known as cytotoxic $\mathrm{T}$ cells. They play an important role in the defense against viruses but also cancer cells. Upon activation they release cytotoxins (e.g., perforin, granzymes, granulysin) into infected or tumorous somatic cells, which eventually leads to the induction of apoptosis. Tumor infiltrating CD8+ cells can induce a potent tumorlytic response, which has been shown for various malignancies [25].

FoxP3+ cells are known as regulatory T-cells (Tregs). The have the ability to suppress effector T-cell function both in a paracrine and cell-cell-contact dependent manner [26]. Tregs are important for the maintenance of immunological tolerance, however, can also dampen antitumor response of the immune system. An expansion of the Treg pool experimentally leads to enhanced vulnerability of carcinogens and worse outcome [27, 28].

The role of TILs has been extensively studied in primary CRC. The inflammatory infiltrate was shown to correlate with the T-stage of primary CRC and even allowed a more precise prognosis on patients' outcome compared to the UICC-TNM staging alone [10]. In the subgroup of rectal cancer patients, the prognostic value of the immune infiltrate (CD3 and CD8) was confirmed and additionally found to be a predictive marker for the response to preoperative chemo-radiotherapy [9]. Based on this data, an international consortium was founded to standardize and implement an adapted staging system taking the immune infiltrate ("Immunoscore") into account [29].

In contrast to the evidence of the role of TILs in primary CRC, sparse data exists on TILs in lung metastases. To the best of our knowledge, by now only two studies have 

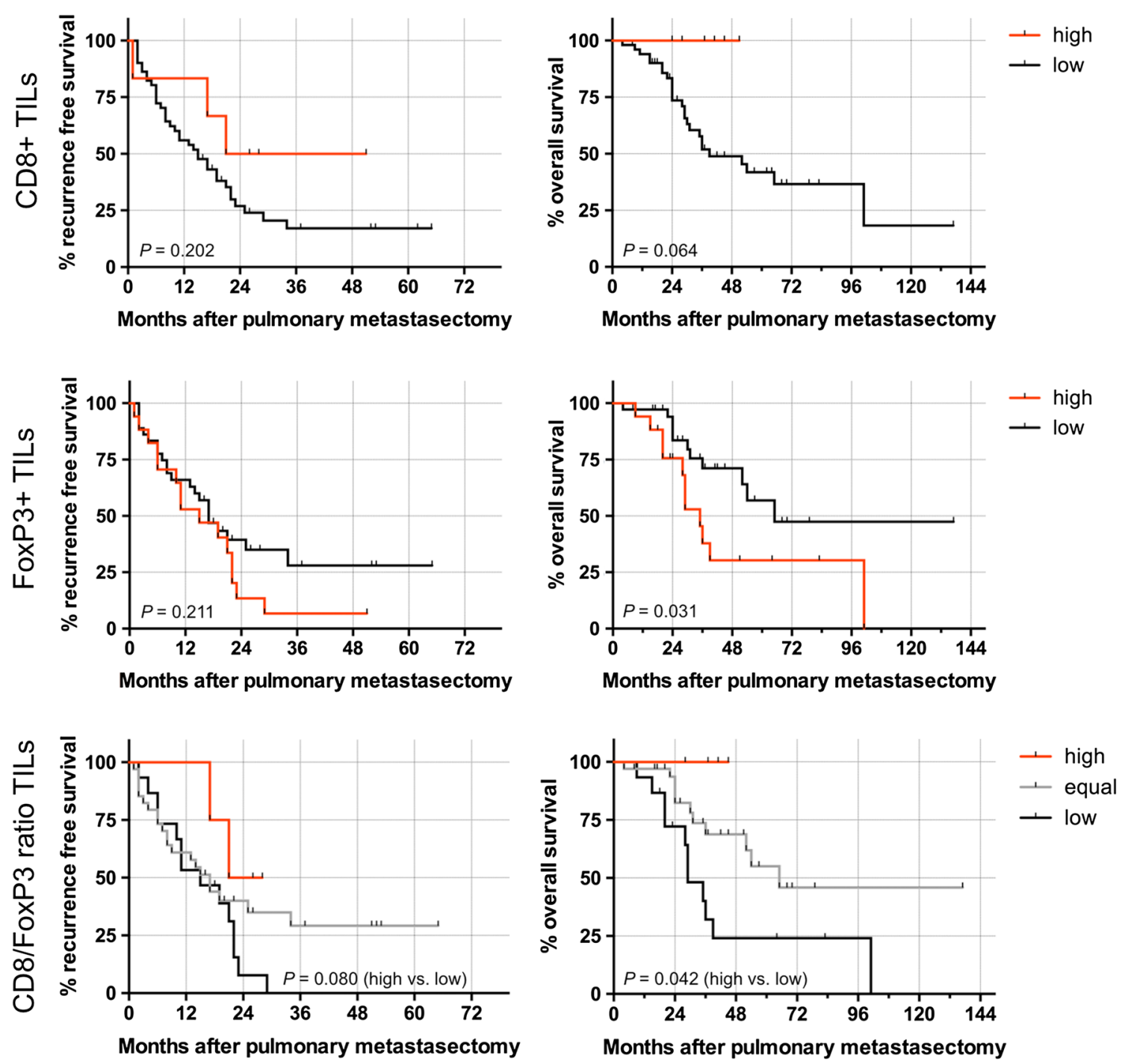

Fig. 2 Kaplan-Meier estimates regarding recurrence-free survival and overall survival of pulmonary metastases dependent on the density of CD8+ and FoxP3+ TILs. Additionally the outcome for the CD8/FoxP3-ratio was calculated

examined TILs in CRC lung metastases. Remark et al. showed in a retrospective cohort with CRC PM that a high density of CD8+ TILs conferred an improved OS $(P=0.039$ in univariate analysis). Interestingly, CD8+ TILs at the tumor center and at the invasive margin had a similar prognostic impact [30]. In another study conducted in a Korean patient cohort with UICC stage IV colon cancer, including 21 patients with PM and 58 with liver metastases, a high CD8+ TILs density in the primary tumor, but not in the metastatic tissue (liver and lung together) had a beneficial impact on OS (log-rank test $P=0.017$ and 0.232 , respectively). The strongest positive prognostic impact was found for high CD45RO+ TILs in the primary and metastatic tissue (log-rank test $P=0.009$ and 0.027 , respectively). The prognostic impact of CD45RO+ TILs in the primary tumor remained significant in a multivariate model (RR 0.108; $95 \%$ CI $0.021-0.546$; $P=0.007)$. Interestingly, a high density of FoxP3+ TILs at the metastatic site was also associated with a favorable prognosis (log rank test $P=0.050$ ) in this study [31]. This is in contrast to most of the published evidence on immunosurveillance in CRC and this finding could not be confirmed in our patient cohort [32,33]. This discrepancy might be explained by the mixture of liver (73\%) and lung metastases $(27 \%)$, which were not separated in the outcome analysis [31]. Another explanation might be the spatial distribution of FoxP3 cells. Salama et al. reported a decreased survival of stage II CRC patients with low content of FoxP3 + T-cells within the tumor (HR 0.65; $95 \%$ CI $0.48-0.89 ; P=0.007)$, whereas the opposite was found when assessing adjacent colonic tissue (HR 1.42; $95 \%$ CI $1.05-1.92 ; \quad P=0.023)$ [11]. The authors 
Table 4 Univariate and multivariate outcome analysis of recurrence-free survival and overall survival after pulmonary metastasectomy (significant values $(P<0.05)$ in bold)

\begin{tabular}{|c|c|c|c|c|c|c|c|c|}
\hline & \multirow{2}{*}{\multicolumn{3}{|c|}{$\frac{\text { Recurrence-free survival }}{\text { Univariate analysis (log-rank) }}$}} & \multicolumn{5}{|c|}{ Overall survival } \\
\hline & & & & \multicolumn{3}{|c|}{ Univariate analysis (log-rank) } & \multicolumn{2}{|c|}{ Multivariate Cox regression } \\
\hline & Months & $\mathrm{HR}(95 \% \mathrm{CI})$ & $P$-value & Months & $\mathrm{HR}(95 \% \mathrm{CI})$ & $P$-value & $\mathrm{HR}(95 \% \mathrm{CI})$ & $P$-value \\
\hline \multicolumn{9}{|l|}{ Sex } \\
\hline Male & 21 & $0.65(0.33-1.19)$ & 0.516 & 54 & $1.30(0.59-2.86)$ & 0.516 & - & - \\
\hline Female & 13.5 & & & 101 & & & - & - \\
\hline \multicolumn{9}{|l|}{ Age (years) } \\
\hline$<64$ & 17 & $1.01(0.54-1.88)$ & 0.974 & 52 & $1.09(0.50-2.40)$ & 0.831 & - & - \\
\hline$\geq 64$ & 15 & & & 65 & & & - & - \\
\hline \multicolumn{9}{|l|}{ Location } \\
\hline Colon & 19 & $0.97(0.52-1.80)$ & 0.917 & 65 & $0.75(0.34-1.62)$ & 0.465 & - & - \\
\hline Rectum & 11 & & & 36 & & & - & - \\
\hline \multicolumn{9}{|l|}{ UICC stage } \\
\hline $\mathrm{I}+\mathrm{II}$ & 17 & $1.14(0.59-2.24)$ & 0.683 & 36 & $1.95(0.88-4.85)$ & 0.099 & - & - \\
\hline $\mathrm{III}+\mathrm{IV}$ & 15 & & & NR & & & - & - \\
\hline Unknown & 3 & & & & & & - & - \\
\hline \multicolumn{9}{|c|}{ Chemotherapy before metastasectomy } \\
\hline Yes & 15 & $1.19(0.57-2.49)$ & 0.648 & 52 & $0.81(0.30-2.09)$ & 0.644 & - & - \\
\hline No & 22 & & & 65 & & & - & - \\
\hline \multicolumn{9}{|c|}{ Chemotherapy after metastasectomy } \\
\hline Yes & 15 & $1.34(0.65-2.72)$ & 0.443 & 39 & $2.08(0.71-4.74)$ & 0.214 & - & - \\
\hline No & 21 & & & NR & & & - & - \\
\hline \multicolumn{9}{|c|}{ Previous liver metastasis } \\
\hline Yes & 9 & $1.99(1.11-4.81)$ & 0.029 & 36 & $1.39(0.61-3.44)$ & 0.414 & - & - \\
\hline No & 21 & & & 54 & & & - & - \\
\hline \multicolumn{9}{|l|}{ DFI } \\
\hline$<36$ & 17 & $1.20(0.62-2.31)$ & 0.600 & 52 & $1.15(0.51-2.62)$ & 0.741 & - & - \\
\hline$\geq 36$ & 17 & & & 65 & & & - & - \\
\hline \multicolumn{9}{|c|}{ No. of nodules } \\
\hline Singular & 17 & $0.93(0.48-1.78)$ & 0.811 & 54 & $0.85(0.37-1.89)$ & 0.681 & - & - \\
\hline Multiple & 16 & & & 36 & & & - & - \\
\hline \multicolumn{9}{|c|}{ CD3+ TILs TC } \\
\hline High & 21 & $0.66(0.35-1.23)$ & 0.199 & 54 & $1.10(0.48-2.53)$ & 0.820 & - & - \\
\hline Low & 15 & & & 52 & & & - & - \\
\hline \multicolumn{9}{|c|}{ CD3+ TILs IM } \\
\hline High & 16 & $1.46(0.77-2.78)$ & 0.254 & 54 & $1.68(0.76-3.89)$ & 0.213 & - & - \\
\hline Low & 19 & & & 101 & & & - & - \\
\hline \multicolumn{9}{|c|}{ CD8+ TILs TC } \\
\hline High & 25 & $0.57(0.29-1.24)$ & 0.181 & 65 & $0.36(0.17-1.27)$ & 0.143 & - & - \\
\hline Low & 15 & & & 39 & & & - & - \\
\hline \multicolumn{9}{|c|}{ CD8+ TILs IM } \\
\hline High & 43 & $0.48(0.23-1.34)$ & 0.202 & NR & $0.00(0.09-1.04)$ & 0.064 & - & - \\
\hline Low & 15 & & & 39 & & & - & - \\
\hline \multicolumn{9}{|c|}{ CD45+ TILs TC } \\
\hline High & 17 & $0.88(0.45-1.66)$ & 0.676 & 65 & $0.78(0.34-1.69)$ & 0.515 & - & - \\
\hline Low & 15 & & & 36 & & & - & - \\
\hline \multicolumn{9}{|c|}{ CD45+ TILs IM } \\
\hline High & 17 & $0.91(0.44-1.84)$ & 0.984 & 54 & $0.79(0.32-1.78)$ & 0.451 & - & - \\
\hline Low & 17 & & & 36 & & & - & - \\
\hline
\end{tabular}


Table 4 continued

\begin{tabular}{|c|c|c|c|c|c|c|c|c|}
\hline & \multirow{2}{*}{\multicolumn{3}{|c|}{$\frac{\text { Recurrence-free survival }}{\text { Univariate analysis (log-rank) }}$}} & \multicolumn{5}{|c|}{ Overall survival } \\
\hline & & & & \multicolumn{3}{|c|}{ Univariate analysis (log-rank) } & \multicolumn{2}{|c|}{ Multivariate Cox regression } \\
\hline & Months & $\mathrm{HR}(95 \% \mathrm{CI})$ & $P$-value & Months & $\mathrm{HR}(95 \% \mathrm{CI})$ & $P$-value & $\mathrm{HR}(95 \% \mathrm{CI})$ & $P$-value \\
\hline \multicolumn{9}{|c|}{ FoxP3+ TILs TC } \\
\hline High & 17 & $0.99(0.52-1.89)$ & 0.982 & 54 & $0.98(0.43-2.27)$ & 0.969 & - & - \\
\hline Low & 15 & & & 52 & & & - & - \\
\hline \multicolumn{9}{|c|}{ FoxP3+ TILs IM } \\
\hline High & 15 & $1.50(0.79-3.16)$ & 0.211 & 35 & $2.40(1.11-6.96)$ & $\mathbf{0 . 0 3 1}$ & $0.61(0.23-1.61)$ & 0.319 \\
\hline Low & 17 & & & 65 & & & & \\
\hline \multicolumn{9}{|l|}{ TLS } \\
\hline Present & 17 & $1.00(0.48-2.10)$ & 0.999 & 52 & $0.65(0.26-1.71)$ & 0.413 & - & - \\
\hline Not present & 15 & & & NR & & & - & - \\
\hline \multicolumn{9}{|l|}{ CD3+ TLS } \\
\hline High & 21 & $1.85(0.36-7.72)$ & 0.525 & 65 & $0.34(0.02-1.70)$ & 0.136 & - & - \\
\hline Low & 19 & & & 52 & & & - & - \\
\hline \multicolumn{9}{|l|}{ CD8+ TLS } \\
\hline High & 21 & $0.58(0.27-1.18)$ & 0.140 & NR & $0.30(0.14-0.79)$ & 0.016 & $4.39(1.25-15.32)$ & 0.020 \\
\hline Low & 11 & & & 35 & & & & \\
\hline \multicolumn{9}{|l|}{ CD45+ TLS } \\
\hline High & 17 & $1.05(0.40-2.80)$ & 0.918 & 65 & $1.03(0.33-3.25)$ & 0.949 & - & - \\
\hline Low & 17 & & & 30 & & & - & - \\
\hline \multicolumn{9}{|l|}{ FoxP3 + TLS } \\
\hline High & 11 & $2.42(0.96-5.59)$ & 0.069 & 39 & $1.50(0.54-4.45)$ & 0.439 & - & - \\
\hline Low & 21 & & & 101 & & & - & - \\
\hline \multicolumn{9}{|c|}{ CD8/FoxP3-ratio IM } \\
\hline Low & 15 & 1 & & 29 & 1 & & - & - \\
\hline Equal & 17 & $0.65(0.30-1.26)$ & 0.197 & 65 & $0.39(0.13-0.83)$ & 0.021 & - & - \\
\hline High & 26 & $0.32(0.12-1.03)$ & 0.080 & NR & $\mathrm{n} / \mathrm{a}$ & 0.042 & - & - \\
\hline \multicolumn{9}{|c|}{ CD8/FoxP3-ratio TLS } \\
\hline Low & 11 & 1 & & 30 & 1 & & - & - \\
\hline Equal & 14 & $0.89(0.32-2.37)$ & 0.804 & 101 & $2.13(0.71-7.60)$ & 0.191 & - & - \\
\hline High & NR & $3.65(1.02-10.96)$ & 0.061 & NR & $6.99(1.28-20.48)$ & 0.027 & - & - \\
\hline
\end{tabular}

$D F I$ disease-free survival to first pulmonary metastasis, $I M$ invasive margin, $N R 50 \%$ recurrence/survival not reached, $T C$ tumor center, $T I L s$ tumor-infiltrating lymphocytes, $T L S$ tertiary lymphoid structure

speculated that FoxP3 + T-regulatory cells might lose their ability to suppress antitumor immunity when they are found within the tumor tissue. Our results suggest differing impact of FoxP3+ TILs depending on their localization. Dense infiltration of FoxP3+ TILs in the infiltration zone was associated with impaired survival, whereas infiltration of FoxP3+ TILs in the tumor center did not alter survival. Moreover, high levels of FoxP3+ TILs at the invasive margin were significantly more common in patients with evidence for lymphatic invasion, which is believed to be a key-determinant of the outcome after pulmonary metastasectomy $[19,31]$. Similar to two recent publications, which described the ratio between CD8+ and FoxP3+ TILs as prognostic factor superior to CD8+ and FoxP3+ TILs alone, we found the CD8/FoxP3- ratio to have a strong impact on OS in our patient cohort [34, 35]. In general, our observations in PMs imply differential roles of TILs dependent on their localization within the metastatic tissue. This has to be taken in consideration when assessing TILs in histological specimen and when defining cut-offs for statistical evaluation.

The present work is, to the best of our knowledge, the first analysis of presence and composition of TLS in CRC lung metastases. We could demonstrate that high levels of CD8+ cells as well as a high CD8/FoxP3-ratio in TLS correlated positively with patients' OS after pulmonary 

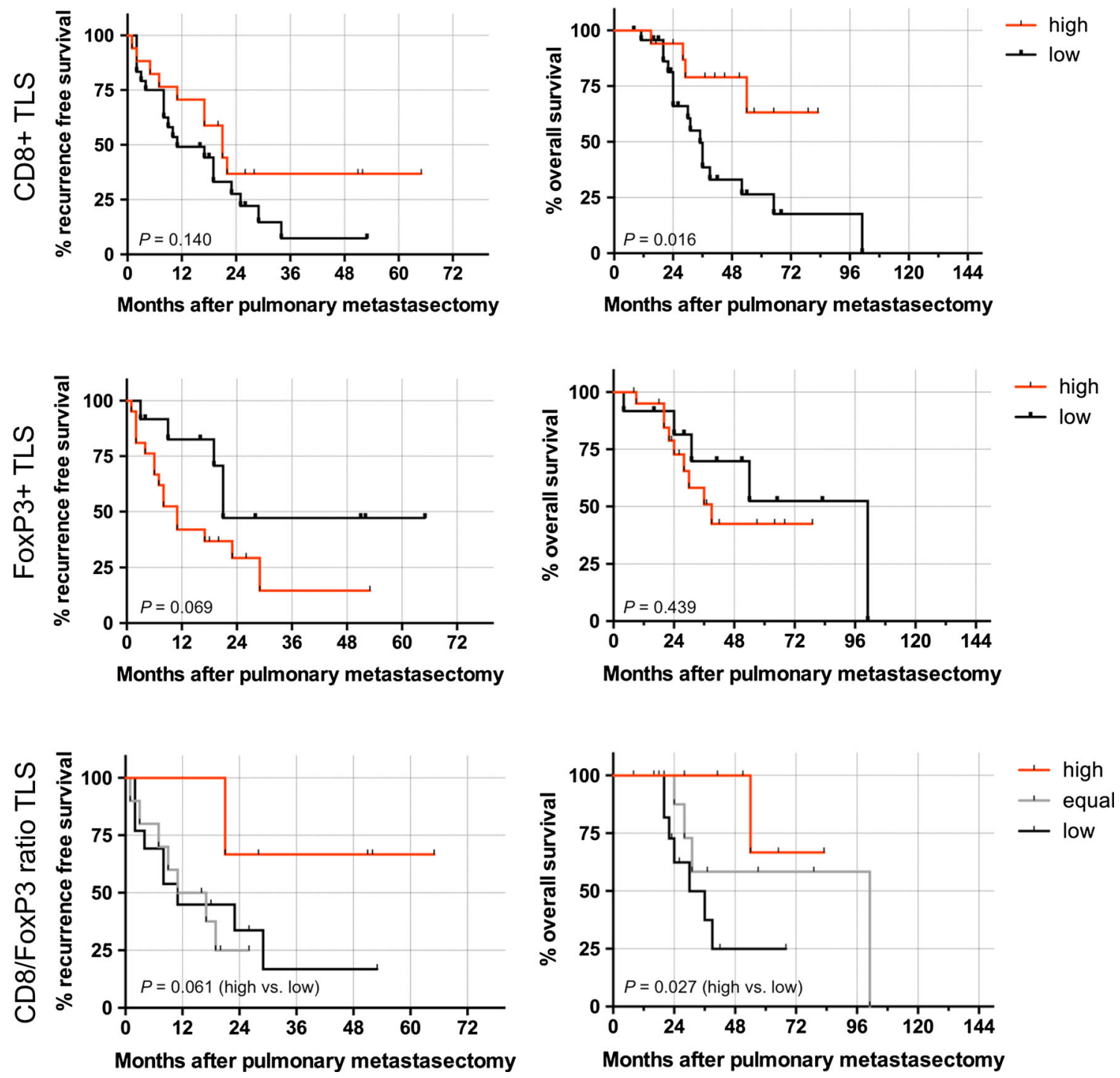

Fig. 3 Kaplan-Meier estimates regarding recurrence-free survival and overall survival of pulmonary metastases dependent on the density of CD8+ and FoxP3 + cells in TLS. Additionally the outcome for the CD8/FoxP3-ratio was calculated

metastasectomy $(P=0.016$ and 0.027 , respectively $)$. Similar to our findings on TILs at the invasive margin, the balance of CD8+ and FoxP3+ in TLS seems to be a crucial factor for the survival of CRC patients with dissemination to the lung. Di Caro et al. found that TLS were present in $78.6 \%$ of stages II and III primary CRC. The presence of TLS was a positive prognosticator only in stage II CRC ( $\log$ rank $P=0.02)$, whereas the prognostic impact was lost in an advanced tumor stage (UICC stage III) [16]. Also in our cohort of stage IV CRC patients, the presence of TLS alone had no impact on recurrence-free and OS. Salama et al. evaluated the distribution of FoxP3 + cells in TLS in stage II colon cancer and described the high density of FoxP3 + cells in TLS as negative prognostic factor of OS $\quad(\mathrm{HR} \quad 4.22 ; 95 \%$ CI $1.49-11.91 ; \quad P=0.007$ in multivariate analysis) [36]. In our cohort, patients with high number of FoxP3+ cells in TLS evidenced a decreased recurrence-free and OS, without reaching the level of significance. Only $7.1 \%$ of the primary CRC samples were rated as TLS positive in our cohort. This might indicate an insufficient immune response at the primary tumor site in patients subsequently developing metastatic disease. However, caution is warranted when interpreting this finding due to the small sample size.

Interestingly, the density of the immune infiltrate in primary tumors did not correlate with the infiltrate in PM. Considering the heterogeneity of tumors, the metastatic spreading of subpopulations of tumor cells of the primary cancer to distant organs might explain the different immuno-phenotype observed in our cohort [37]. In 
general, lung metastases appear to consist of more immunogenic tumor cells compared to the primary tumor site, since the density of CD3+, CD8+ and CD45+ TIL increased during progression of disease. Another possible explanation for this observation might be the unique immunological state of the lung. It is constantly exposed to environmental pathogens and therefor rich in residing immune cells.

Our findings suggest that patients with CRC lung metastases is a heterogeneous group regarding the tumor biology. This might have implications on the management of these patients. Histological evidence for an aggressive tumor biology might have consequences regarding the offered adjuvant chemotherapy, tumor surveillance strategies and a possible future re-metastasectomy.

There are several limitations to this study. First, no uniform protocol for pseudo-neoadjuvant and pseudo-adjuvant chemotherapeutic regimens was applied. According to the preferences of the referring oncologist, different schemes of chemotherapy were administered to the patients. As chemotherapeutic agents might contribute to the immune-editing of tumors, this is a possible confounder of this study. However we could not find a significant association between administration of chemotherapy before metastasectomy and the immune infiltrate in PMs. Moreover, the history of chemotherapy before metastasectomy did not affect the outcome after lung metastasectomy. Second, patients eligible for surgery are a subgroup and do not represent the whole population of patients with lung metastases from CRC. Thus, our findings might not be extrapolated to all patients with CRC PMs due to this selection bias. Last, although this is currently the biggest cohort of patients with CRC PM in which the CD3+, CD8+, CD45RO+ and FoxP3+ TILs and TLS was assessed, the study might still be underpowered. A multiinstitutional study with a considerable sample size will be necessary to further clarify the prognostic value of TILs and TLS in CRC lung metastases.

In summary, this is the first structured analysis of $\mathrm{CD} 3+, \mathrm{CD} 8+, \mathrm{CD} 45 \mathrm{RO}+$ and FoxP3+ TILs and TLS in the setting of curative pulmonary metastasectomy, including more than 50 patients with CRC lung metastases. Our results suggest that especially the balance of effector CD8+ and regulatory FoxP3+ TILs play a crucial role during immune-editing of metastatic CRC and thus, predicting outcome of patients after pulmonary metastasectomy with curative intent.

Acknowledgments This study was supported by a Research Grant (\#15880) provided by the Austrian Federal Bank (OeNB), by a Grant provided by Initiative Krebsforschung (Project Title "Tumorimmunologie von Hirnmetastasen") and by the Christian Doppler Laboratory for Cardiac and Thoracic Diagnosis and Regeneration.
Open Access This article is distributed under the terms of the Creative Commons Attribution 4.0 International License (http://crea tivecommons.org/licenses/by/4.0/), which permits unrestricted use, distribution, and reproduction in any medium, provided you give appropriate credit to the original author(s) and the source, provide a link to the Creative Commons license, and indicate if changes were made.

\section{References}

1. Mitry E et al (2010) Epidemiology, management and prognosis of colorectal cancer with lung metastases: a 30-year populationbased study. Gut 59(10):1383-1388

2. Erstad DJ, Tumusiime G, Cusack JC Jr (2015) Prognostic and predictive biomarkers in colorectal cancer: implications for the clinical surgeon. Ann Surg Oncol 22(11):3433-3450

3. Renaud S, Falcoz PE (2015) How can we identify new biomarkers for patients with for lung metastasectomy in colorectal cancer. Future Oncol 11(15):2109-2111

4. Owusu BY et al (2015) Prognostic and predictive significance of stromal fibroblasts and macrophages in colon cancer. Biomark Cancer 7(Suppl 1):29-37

5. Schweiger $\mathrm{T}$ et al (2014) Prognostic factors in pulmonary metastasectomy: spotlight on molecular and radiological markers. Eur J Cardiothorac Surg 45(3):408-416

6. Schweiger T et al (2015) Stromal expression of heat-shock protein 27 is associated with worse clinical outcome in patients with colorectal cancer lung metastases. PLoS One 10(3):e0120724

7. Junttila MR, de Sauvage FJ (2013) Influence of tumour microenvironment heterogeneity on therapeutic response. Nature 501(7467):346-354

8. Hanahan D, Weinberg RA (2011) Hallmarks of cancer: the next generation. Cell 144(5):646-674

9. Anitei MG et al (2014) Prognostic and predictive values of the immunoscore in patients with rectal cancer. Clin Cancer Res 20(7):1891-1899

10. Mlecnik B et al (2011) Histopathologic-based prognostic factors of colorectal cancers are associated with the state of the local immune reaction. J Clin Oncol 29(6):610-618

11. Salama $P$ et al (2009) Tumor-infiltrating FOXP3 + T regulatory cells show strong prognostic significance in colorectal cancer. J Clin Oncol 27(2):186-192

12. Donnem T et al (2015) Stromal CD8+ T-cell density-a promising supplement to TNM staging in non-small cell lung cancer. Clin Cancer Res 21(11):2635-2643

13. Miyashita $M$ et al (2015) Prognostic significance of tumor-infiltrating CD8+ and FOXP3+ lymphocytes in residual tumors and alterations in these parameters after neoadjuvant chemotherapy in triple-negative breast cancer: a retrospective multicenter study. Breast Cancer Res 17(1):124

14. Giraldo NA et al (2015) Orchestration and prognostic significance of immune checkpoints in the microenvironment of primary and metastatic renal cell cancer. Clin Cancer Res 21(13):3031-3040

15. McMullen TP et al (2010) Survival in rectal cancer is predicted by $\mathrm{T}$ cell infiltration of tumour-associated lymphoid nodules. Clin Exp Immunol 161(1):81-88

16. Di Caro G et al (2014) Occurrence of tertiary lymphoid tissue is associated with T-cell infiltration and predicts better prognosis in early-stage colorectal cancers. Clin Cancer Res 20(8):2147-2158

17. Halama $\mathrm{N}$ et al (2013) Hepatic metastases of colorectal cancer are rather homogeneous but differ from primary lesions in terms of immune cell infiltration. Oncoimmunology 2(4):e24116 
18. Keim S et al (2012) Sequential metastases of colorectal cancer: immunophenotypes and spatial distributions of infiltrating immune cells in relation to time and treatments. Oncoimmunology 1(5):593-599

19. Schweiger $T$ et al (2015) Increased lymphangiogenesis in lung metastases from colorectal cancer is associated with early lymph node recurrence and decreased overall survival. Clin Exp Metastasis 33(2):133-141

20. Berghoff AS et al (2016) Density of tumor-infiltrating lymphocytes correlates with extent of brain edema and overall survival time in patients with brain metastases. Oncoimmunology 5(1): e1057388

21. Berghoff AS et al (2013) Characterization of the inflammatory response to solid cancer metastases in the human brain. Clin Exp Metastasis 30(1):69-81

22. Menon AG et al (2004) Immune system and prognosis in colorectal cancer: a detailed immunohistochemical analysis. Lab Investig 84(4):493-501

23. Koelzer VH et al (2014) CD8/CD45RO T-cell infiltration in endoscopic biopsies of colorectal cancer predicts nodal metastasis and survival. J Transl Med 12:81

24. Bender R, Lange S (2001) Adjusting for multiple testing-when and how? J Clin Epidemiol 54(4):343-349

25. Nesbeth Y, Conejo-Garcia JR (2010) Harnessing the effect of adoptively transferred tumor-reactive $\mathrm{T}$ cells on endogenous (host-derived) antitumor immunity. Clin Dev Immunol 2010: 139304

26. Zou W (2006) Regulatory $\mathrm{T}$ cells, tumour immunity and immunotherapy. Nat Rev Immunol 6(4):295-307

27. Nishikawa $\mathrm{H}$ et al (2005) Accelerated chemically induced tumor development mediated by $\mathrm{CD} 4+\mathrm{CD} 25+$ regulatory $\mathrm{T}$ cells in wild-type hosts. Proc Natl Acad Sci USA 102(26):9253-9257
28. Nishikawa $\mathrm{H}$ et al (2003) $\mathrm{CD} 4+\mathrm{CD} 25+\mathrm{T}$ cells responding to serologically defined autoantigens suppress antitumor immune responses. Proc Natl Acad Sci USA 100(19):10902-10906

29. Galon $\mathrm{J}$ et al (2014) Towards the introduction of the 'Immunoscore' in the classification of malignant tumours. J Pathol 232(2):199-209

30. Remark R et al (2013) Characteristics and clinical impacts of the immune environments in colorectal and renal cell carcinoma lung metastases: influence of tumor origin. Clin Cancer Res 19(15): 4079-4091

31. Lee WS et al (2013) Clinical impact of tumor-infiltrating lymphocytes for survival in curatively resected stage IV colon cancer with isolated liver or lung metastasis. Ann Surg Oncol 20(2): 697-702

32. Lee WS et al (2010) Clinical impact of tumor-infiltrating lymphocytes for survival in stage II colon cancer. Cancer 116(22): $5188-5199$

33. Hanke $\mathrm{T}$ et al (2015) High intratumoral FOXP3(+) T regulatory cell (Tregs) density is an independent good prognosticator in nodal negative colorectal cancer. Int $\mathbf{J}$ Clin Exp Pathol 8(7): $8227-8235$

34. Zeestraten EC et al (2013) FoxP3- and CD8-positive infiltrating immune cells together determine clinical outcome in colorectal cancer. Cancer Microenviron 6(1):31-39

35. Suzuki $H$ et al (2010) Intratumoral CD8(+) T/FOXP3 (+) cell ratio is a predictive marker for survival in patients with colorectal cancer. Cancer Immunol Immunother 59(5):653-661

36. Salama P et al (2012) FOXP3 + cell density in lymphoid follicles from histologically normal mucosa is a strong prognostic factor in early stage colon cancer. Cancer Immunol Immunother 61(8):1183-1190

37. Al-Hajj M et al (2003) Prospective identification of tumorigenic breast cancer cells. Proc Natl Acad Sci USA 100(7):3983-3988 